\title{
Germanica
}

\section{Barbara Frischmuth et la mise en question du discours éducatif patriarcal}

Barbara Frischmuth und die Infragestellung des patriarchalischen

Erziehungsdiskurses.

Barbara Frischmuth and the questioning of patriarchal discurse.

\section{Hélène Barrière}

\section{(2) OpenEdition}

Journals

Édition électronique

URL : http://journals.openedition.org/germanica/1054

DOI : 10.4000/germanica.1054

ISSN : 2107-0784

Éditeur

Université de Lille

\section{Édition imprimée}

Date de publication : 30 juin 2010

Pagination : 107-124

ISBN : 9782913857254

ISSN : 0984-2632

\section{Référence électronique}

Hélène Barrière, «Barbara Frischmuth et la mise en question du discours éducatif patriarcal », Germanica [En ligne], 46 | 2010, mis en ligne le 01 juin 2012, consulté le 06 octobre 2020. URL : http:// journals.openedition.org/germanica/1054; DOI : https://doi.org/10.4000/germanica.1054

Ce document a été généré automatiquement le 6 octobre 2020.

(c) Tous droits réservés 


\title{
Barbara Frischmuth et la mise en question du discours éducatif patriarcal
}

\author{
Barbara Frischmuth und die Infragestellung des patriarchalischen \\ Erziehungsdiskurses. \\ Barbara Frischmuth and the questioning of patriarchal discurse.
}

\section{Hélène Barrière}

1 «J'ai rêvé que j'étais une lumière - et que quelqu'un la mettait sous son boisseau » ${ }^{1}$. Ainsi s'ouvre le septième des quatorze chapitres qui composent Die Klosterschule (Chez les sœurs), premier roman publié en 1968 par une jeune femme de vingt-sept ans ${ }^{2}$. Par sa position centrale et son titre, cette section du texte baptisée « Le rêve » requiert une attention particulière : selon une dialectique bien connue s'y révèle, par le jeu d'une association inédite de bribes de réalité, la vérité cachée de cette dernière. Un songe nocturne de la narratrice, élève d'un pensionnat catholique autrichien, s'empare pour le distordre du texte biblique ${ }^{3}$, et met ainsi au jour la vraie nature de l'éducation reçue : au lieu d'aider les élèves à rayonner, à reconnaître et à déployer les virtualités de leurs jeunes vies, on les place sous un éteignoir, on brise l'élan de leur esprit et de leur sensibilité. Cette mutilation qui tranche dans le vif des possibles est, comme l'indique le possessif «son", une annexion, mise en œuvre à l'aide d'un système coercitif bien défini. Au sein de Die Klosterschule, le chapitre du rêve constitue l'échappée qui dit le plus nettement le propos de l'ouvrage: la mise en question de la soumission aux structures patriarcales inculquée dans les internats catholiques de filles. Le reste du texte est régi par un mécanisme moins apparent.

2 Nous nous proposons d'observer le fonctionnement de ce dispositif narratif, de dégager les enjeux de sa subtilité et de tenter d'estimer, par l'étude de la réception de Die Klosterschule, son efficacité. Les pistes présentes dans le premier roman de B. Frischmuth nous permettront ensuite d'esquisser les formes ultérieures d'un 
questionnement soucieux de dilater une réalité donnée pour intangible, de l'élargir à la prise en compte de toutes les composantes d'une vie féminine.

\section{Modalités et teneur de la critique dans Die Klosterschule}

3 Lorsque parait Die Klosterschule, B. Frischmuth est encore inconnue du grand public, auquel cet écrit la révèle. Mais ses débuts en littérature remontent à 1960 où, à dix-neuf ans, elle a été membre fondateur du Forum Stadtpark de Graz, aux côtés d'un Alfred Kolleritsch ou d'un Peter Handke. Elle a publié des poèmes et de petits textes de prose dans la revue du groupe, manuskripte. Cette appartenance inscrit ses premières productions dans une démarche particulière, ainsi formulée par K. Bartsch :

Unhintergehbar ist [...] den «Grazer» Autoren [...] die Erfahrung, daß experimentelle Einstellung zur Sprache eingefahrene, verordnete Weisen der Wirklichkeitsaneignung und Wirklichkeitsinterpretation, Absolutheitsansprüche geschlossener Ideologien, naiven Sprachrealismus zu unterlaufen vermag und daß diese Art von Subversivität eine eigene, wenn auch sehr vermittelte Form öffentlichen, ja politischen Handelns darstellt ${ }^{4}$.

Le Forum Stadtpark est né en réaction à un refus institutionnel de toute innovation artistique lié à la situation singulière de l'Autriche d'après-guerre. Exonérée par le préambule du Traité d'État de 1955 de toute responsabilité dans la Seconde Guerre mondiale, elle se pose en victime de l'Anschluss: reconstruction consensuelle et fréquentation touristique sont les maîtres mots d'une politique tout entière placée sous le signe de la Vergangenheitsverdrängung. En termes culturels, cette dernière se traduit par la volonté de restaurer envers et contre tout, au mépris de l'ineffaçable césure du nazisme, la continuité d'une tradition. Il s'agit de célébrer les valeurs éternelles et l'Autriche héritière de l'Empire romain et du Saint-Empire romain germanique, dont l'aptitude millénaire à concilier les contraires pourrait faire référence en Europe. Une Autriche dont "l'idée», déjà vidée de son contenu proprement politique et spiritualisée dans les écrits de Hofmannsthal $^{5}$, est proposée comme modèle identificatoire, comme ciment de l'édification nationale ${ }^{6}$. Dans le cadre étroit de la province autrichienne, cette atmosphère est plus pesante encore que dans la capitale où parvient à émerger dès les années cinquante, avec le "Groupe de Vienne ", une avant-garde qui se voue à l'expérimentation langagière. À Graz, jusqu'au début des années soixante, écrivains et universitaires compromis sous le Troisième Reich tiennent le haut du pavé et dictent sans appel la norme artistique. La plus haute distinction littéraire de Styrie, le Prix Peter Rosegger, est décernée à des auteurs qui ont manifesté leur sympathie pour le nazisme ${ }^{7}$. C'est ce joug de la réaction que les auteurs du Forum Stadtpark entendent secouer. S'ils se proclament d'emblée "avantgarde inconditionnelle $»^{8}$, c'est plus par provocation que par véritable rigueur programmatique 9 . Il n'en demeure pas moins que le Forum Stadtpark ouvre grandes ses portes à l'avant-garde et noue avec le Groupe de Vienne de fructueux échanges: il accueille dans la revue manuskripte les textes des Viennois, leur offrant une tribune qui leur permet de sortir de la confidentialité ; ceux-ci familiarisent en retour leurs jeunes collègues avec les productions des avant-gardes «historiques » du début du $\mathrm{xx}^{\mathrm{e}}$ siècle (futurisme, dadaïsme, expressionnisme) ainsi qu'avec leur propre travail sur la langue, nourri de la critique du langage telle que l'a menée, en particulier, Ludwig Wittgenstein dans son Tractatus logico-philosophicus (1921). Le Groupe de Vienne et, par son 
entremise, la réflexion conduite par Wittgenstein exercent ainsi sur les premiers textes des écrivains du Forum Stadtpark une indéniable influence. À leurs yeux, le lieu de l'assujettissement de l'individu est le langage, congruent à une forme de société qui se perpétue à travers lui. Dès le plus jeune âge, le carcan des formules convenues est imposé à l'individu pour le contraindre à rester dans le rang. Ce langage dévoyé, réduit à un instrument de coercition, s'interpose entre l'individu et ses expériences, lui interdisant de les penser. Seul le travail sur la langue envisagée comme matériau, l'expérimentation qui démasque les idéologies et déploie des constellations expressives inédites peut éventuellement desserrer l'étau.

5 L'écriture de Die Klosterschule fait écho à ces analyses. Le procédé qu'elle privilégie est celui du montage, qui, par excellence, s'inscrit en faux contre l'idéologie religieuse, sociale et esthétique d'un tout harmonieux et immuable sur laquelle se fonde la littérature traditionaliste en Autriche jusque dans les années soixante. L'organisation de l'ouvrage met en évidence l'étroitesse et le caractère parcellaire (l'ignorance est mère de soumission) de la vision du monde proposée aux jeunes filles du pensionnat. Les quatorze sections ${ }^{10}$ ne s'ordonnent pas en une continuité narrative, mais juxtaposent des bribes de quotidien dont l'agencement participe de la logique dénonciatrice du texte et nullement de la chronologie. "Le rêve " central est ainsi flanqué de "La leçon de bienséance " et du "Bonheur». Le premier de ces deux chapitres se compose d'un montage de citations empruntées au discours des sœurs sur la manière dont il faut en user - c'est-à-dire ruser - avec les hommes pour parvenir à convoler en justes noces avec l'un d'eux. Le désir masculin savamment entretenu, dosé et laissé inassouvi jusqu'au mariage est présenté, si l'on peut dire, comme une arme "maitresse", tandis que les mots "amour " et "bonheur " sont absents des formules reproduites. La relation au sexe masculin y revêt des allures de parcours du combattant, dont l'enjeu, si l'on y regarde de près, n'est pas à la hauteur de la mise : les femmes ne gagnent que le droit d'accomplir leurs devoirs à la place qui leur est prétendument assignée de toute éternité dans la société. Faisant d'une pierre deux coups, la rhétorique des sœurs cimente une réalité donnée pour incontournable et la brandit en même temps comme épouvantail, afin de susciter des vocations religieuses :

Bevor ihr aber all dies auf euch nehmt, bevor ihr also ins Leben geht, um dort den euch zugewiesenen Platz einzunehmen und die euch bestimmten Aufgaben zu erfüllen, sollt ihr noch mehrmals und gut erwägen, daß euch auch ein anderer Weg offensteht. [...] Wählt ihr ihn, so wählt ihr einen Stand, der nicht nur den Vorteil hat, euch schon zu Lebzeiten in [Gottes] unmittelbare Nähe zu bringen, sondern von euch auch die obengenannten Sorgen und Nöte fernzuhalten ${ }^{11}$.

6 La succession des titres de sections suggère que celle nommée "Le bonheur » nous instruira sur les aspirations véritables des jeunes filles, articulées grâce au « rêve » qui la précède. Mais celui-ci, nous le verrons, demeure entravé, impuissant à recomposer le monde, à le soustraire à l'emprise du discours éducatif. La stratégie de dérision joue ici de l'inadéquation du terme "bonheur " (précédé de l'article défini, il semble appeler l'évocation du bonheur en soi) à la réalité désignée : l'ingestion compensatoire, la consommation immodérée de gâteries envoyées par la famille pour "digérer" une punition ou une injustice.

Nicht immer bereiten wir uns das Vergnügen zu essen. Es muß einen Grund dafür geben. Ein Mißgeschick zum Beispiel, das uns ereilt, veranlaßt uns zum Essen. Wenn wir wirklich essen, tun wir das, um glücklich zu werden. [...] Ein sicheres Glück, das wir uns in traurigen Zeiten nicht vorenthalten ${ }^{12}$. 
7 Entre les murs du pensionnat, «le bonheur» ne saurait être qu'un vain mot. Sa définition dévoile comment, chez les sœurs, le langage forme écran devant l'expérience qu'il est censé dire. L'univers imposé aux jeunes filles est une construction langagière qui masque le chatoiement de la vie, réduit le réel à des proportions dérisoires, l'ampute des virtualités du féminin. L'éclatement du texte en quatorze morceaux renvoie en sourdine, sans quitter le registre du religieux, à cette mutilation : quatorze n'est-il pas le nombre des stations du chemin de croix ${ }^{13}$ ? La seule logique qui préside à l'ordonnance des chapitres est donc celle d'une accumulation de sévices psychologiques aboutissant à la mort, entendons à l'anéantissement de toute individualité, broyée par l'endoctrinement. Dès l'incipit, Die Klosterschule noie le «je » dans le « nous » de la litanie :

Wir, Angehörige der katholischen Jungschar, Zöglinge des Klosters, Schülerinnen der Ober- und Unterstufe, beten täglich und gerne: das Morgengebet vor Tagesbeginn, das Schulgebet vor Schulbeginn, das Schlußgebet nach Unterrichtsschluß, das Tischgebet vor und nach Tisch, das Studiengebet vor und nach dem Studium, das Abendgebet am Abend [... ${ }^{14}$.

Le premier chapitre compte en tout quatre phrases. Le constat liminaire comprend trente-trois lignes, la description des attitudes de prière neuf, celle des vertus de cette dernière quarante-quatre, tandis que la concision de l'assertion finale souligne l'ampleur des efforts fournis en regard de l'incertitude du résultat : "Et c'est pourquoi nous prions jusqu'à ce que nous soyons secourues ${ }^{15}$. Le "je » ne parvient pas à émerger dans ces pages où le sujet, contraint de psalmodier à perdre haleine, s'asphyxie. K. Zeyringer note que le titre du chapitre suivant semble une promesse d'échappée individuelle, aussitôt déçue :

Das zweite Kapitel kündigt in der Überschrift eine Öffnung an (« Spazierengehen »), die aber gleich im ersten Satz zurückgenommen wird : «Sich anschließen oder sich ausschließen : als ob wir die Wahl hätten ». Erst nachdem das Spazierritual, die "Gehordnung ", dargestellt ist, darf erstmals ein schüchternes "Ich", eingeklemmt in der Gemeinschaft, auftreten ${ }^{16}$

9 Absent de la moitié des chapitres, le « je » n'apparaît dans les autres que par effraction, dans la parenthèse d'un manquement à la règle ou d'une situation (maladie, rêve) qui le soustrait l'espace d'un instant à la communauté. Th. Rothschild observe que, si les sœurs ont toutes un nom, la narratrice demeure anonyme - privée d'individualité ${ }^{17}$.

Précisément: le «je» est ici un «je » narratif, et ce que le pensionnat refuse aux femmes, c'est d'inventer leur place non seulement dans la société, mais dans l'art. L'une des contraintes qui pèse sur les élèves est, en effet, l'interdiction de raconter des histoires dans le noir, après l'extinction des lumières du dortoir ${ }^{18}$. Or, le récit nocturne possède dans l'univers de $B$. Frischmuth une signification bien particulière, incarnée par Schéhérazade. L'écrivaine évoque cette figure des Mille et une nuits dans les conférences de poétique qu'elle tient en 1990 à l'Université de Munich. L'art de conter déployé par Schéhérazade, qui s'offre d'elle-même à épouser le roi Shâhriyâr pour « délivre[r] les jeunes femmes [de ses] griffes ${ }^{19}$, n'est nullement, pour B. Frischmuth, la ruse du faible, mais un défi de l'art à la mort, une manière de triompher de celle-ci en la subjuguant par la puissance de l'évocation :

Nur erzählend kann sie am Leben bleiben. «Ich erzähle, also bin ich ». Es ist ein Erzählen wider den Tod [...]. Dennoch deutet die Motivation der Schehrezâd nicht auf eine teleologische Ausrichtung des Erzählens hin, das wie alle Kunst den Zweck [...] in sich selbst hat. Schehrezâds Erzählen ist vielmehr so stark, daß es sich sogar gegen den Tod setzt $[. . .]^{20}$. 
11 La narratrice de Die Klosterschule est, en quelque sorte, une Schéhérazade muselée, à qui l'on s'ingénie à désapprendre le désir de conter pour éviter qu'elle n'en vienne à conjuguer le récit au féminin et n'échappe ainsi à l'ordre patriarcal travesti en destin. Citation et montage évoquent la manière dont l'imagination est impitoyablement bannie de l'univers du pensionnat.

12 Mais, avant tout, ces procédés d'écriture participent de ce que L. Irigaray définira plus tard, dans les années soixante-dix, comme la stratégie du mimétisme. Pour résoudre «la question de l'articulation du sexe féminin dans le discours ${ }^{21}$, il faut, dit-elle, entreprendre de détruire le fonctionnement discursif. Et de préciser :

Il n'est, dans un premier temps, peut-être qu'un seul «chemin ", celui qui est historiquement assigné au féminin : le mimétisme. Il s'agit d'assumer, délibérément, ce rôle. Ce qui est déjà retourner en affirmation une subordination, et, de ce fait, commencer à la déjouer. [...] Jouer de la mimésis, c'est donc, pour une femme, tenter de retrouver le lieu de son exploitation par le discours, sans s'y laisser simplement réduire. C'est se resoumettre [...] à des "idées ", notamment d'elle, élaborées dans/ par une logique masculine, mais pour faire "apparaître », par un effet de répétition ludique, ce qui devait rester occulté : le recouvrement d'une possible opération du féminin dans le langage. C'est aussi « dévoiler » le fait que, si les femmes miment si bien, c'est qu'elles ne se résorbent pas simplement dans cette fonction. Elles restent aussi ailleurs [...] $]^{22}$.

Die Klosterschule reprend en écho, différemment agencées, corsées d'un soupçon d'ironie ou d'un grossissement imperceptible du trait, les formules du discours éducatif en vigueur dans les pensionnats catholiques de jeunes filles, afin de le subvertir. Mais si certains interprètes saisissent parfaitement l'enjeu de l'ouvrage et notent l'adéquation de l'option narrative et du dessein ${ }^{23}$, d'autres auraient souhaité des choix plus lisiblement incisifs, tel U. Stamer qui déclare que B. Frischmuth «n'a pas eu le courage de la parodie $»^{24}$. Ce jugement manifeste l'incompréhension de la démarche adoptée par l'auteure, qui aurait manqué de distance par rapport au sujet, négligé de laisser l'imagination féconder l'écriture, limité celle-ci à une imitation qui en signe l'impuissance :

Vieles bleibt zu kontemplativ, zu wenig kritisch, im Prozeß der Verbalisierung zu unreflektiert. [...] Der behutsame Vorwurf, die verhaltene Anklage werden schon durch die kommentarlose Manier des nüchternen Referierens unüberhörbar. [...] $[\mathrm{N}]$ icht selten aber bleibt der - zweifellos erforderliche - Freiraum zur Assoziation ungenutzt, weil die erzählerische Absicht zwar erkennbar ist, die phantasieoffene Anregung jedoch fehlt. [...] [D]urch die Imitation vorgegebener Sprachmuster wird über Regeln und Verbote noch keine Herrschaft erlangt ${ }^{25}$.

\section{Limites de la mimésis}

Il convient donc de se demander si les modalités de la dénonciation sont de nature à brouiller le propos. Th. Rothschild fournit l'exemple d'une analyse incertaine de la charge de révolte dont le texte doit être crédité. Il ne voit pointer une velléité de rébellion de la narratrice que dans le dernier chapitre, une lettre qu'elle adresse à l'une de ses condisciples qui a quitté l'internat pour une école publique et perdu la foi ${ }^{26}$. La missive établit un contact particulier avec le dehors, instaure la possibilité d'un commentaire de l'expérience commune formulé à l'aide de critères en partie extérieurs. La narratrice se projette dans un avenir où elle aura, elle aussi, quitté le 
pensionnat. Mais elle sait bien qu'elle n'en aura pas pour autant échappé aux sœurs, dont le patient travail de formatage va orienter sa vie entière :

Th. Rothschild commente :

Auch hier signalisiert noch kein eindeutiges Entscheidungskriterium, ob die Ansichten über die Gottgewolltheit von Gewalt und Obrigkeit von der Briefschreiberin geteilt werden, oder ob sie sie ironisch, aus der Distanz zitiert. Der Text lässt beide Lesarten $\mathrm{zu}^{28}$.

Une telle remarque nous semble démentie, par exemple, par le début du deuxième chapitre cité plus haut ${ }^{29}$. Les manquements à la règle qui composent, exception faite des lignes consacrées au rêve, l'espace narratif dévolu au «je» par opposition au «nous » infirment aussi la thèse d'une adhésion de la narratrice au fonctionnement du pensionnat. Toutefois, la perplexité dont témoigne l'étude de Th. Rothschild n'est pas un cas isolé. Elle s'exprime déjà dans un certain nombre de recensions publiées au moment de la parution de Die Klosterschule, puis de sa première réédition en 1978. Elle participe d'une confusion favorisée par la technique de la citation et celle de la narration simultanée. La première maintient sur la "paternité" de l'énoncé un doute permanent; la seconde, selon un mécanisme décrit par G. Genette, fait basculer le récit vers le discours ${ }^{30}$. Dans les commentaires s'opèrent donc des identifications illicites, mises au jour par les contradictions qu'elles engendrent. L'opinion de la narratrice serait, par exemple, indiscernable du discours collectif placé dans sa bouche, procédé jugé monocorde et lassant; mais l'on reproche ensuite à B. Frischmuth la fin du texte qui, en regard de l'expression voilée d'une subjectivité à l'œuvre dans les chapitres précédents, dirait trop clairement l'asservissement $\mathrm{du}$ " je $»^{31}$. Au détour d'une phrase, se produit un glissement de la narratrice à l'auteure : l'on dénie au discours de la première le tour parodique dont la maladresse est reprochée quelques lignes plus loin à B. Frischmuth ${ }^{32}$; l'on cherche dans l'hésitation supposée de cette dernière à renier catégoriquement les préceptes de la religion la raison d'une attaque trop empreinte de retenue $^{33}$. Nous touchons là à une autre restriction apportée au propos de B. Frischmuth: celle qui se fonde sur la coloration autobiographique de Die Klosterschule. Originaire d'Altaussee, villégiature au bord d'un lac entre la Styrie et la Haute-Autriche, B. Frischmuth a été, de dix à quatorze ans, élève des sœurs de la Croix à Gmunden, sur les rives du Traunsee. Une condition vécue d'autant plus durement que son père était tombé à Kiev en 1943 et que sa mère, ruinée, ne parvenait pas toujours à payer la pension à temps ${ }^{34}$. B. Frischmuth n'aurait inventé aucun des détails mentionnés dans son texte ${ }^{35}$. Pour certains interprètes, le statut de souvenir personnel signerait la nature singulière de l'expérience évoquée et le caractère subjectif de la perception de celle-ci ${ }^{36}$. R. Priessnitz et $\mathrm{M}$. Rausch, qui font grief aux premières productions des auteurs du Forum Stadtpark de n'être que "post-expérimentales", c'est-à-dire simple synthèse commercialement réussie entre expérimentations de l'avant-garde et modes de narration conventionnels, se situent dans une logique voisine. L'usage de la citation, dans Die Klosterschule, ne produit selon eux qu'un effet d'authenticité (proche de l'« effet de réel » décrit par R. Barthes ${ }^{37}$ ) et n'a rien d'une tactique subversive :

das der montage ansonsten eigenständige (kontrastierende, offene) versagt sich dem hier durchgeführten verfahren und ordnet sich dem übrigen erzählten unter, geht in dieses auf. [...] die in der prosa der frischmuth vorgeformten teile haben dort eher die funktion einer vermittlerrolle zwischen dem erlebten (erfahrenen) und dem auf ihm basierenden fiktiven (dem, was die arbeit zu einer erzählung macht). die zitateinheiten [...] legitimieren deren anspruch auf authentizität, rechtfertigen jene 
gleichsam. die subjektive zutat, das [...] zitat als beweisstück, argumentationsbrücke, orientierungsbehelf ${ }^{38}$. B. Frischmuth a reçu un mauvais accueil. Il n'en est rien: dès 1968, la très large adhésion de la critique ouvre au contraire à la jeune femme, par le biais de tournées de lectures, d'émissions radiophoniques ou d'invitations à des rencontres d'écrivains, la voie de la notoriété. Toutefois, les analyses mitigées que nous avons observées nous ont permis de dégager plus aisément le dénominateur commun des malentendus: ils ne rendent pas pleinement justice à la texture d'une écriture résolument, mais finement subversive. Un affadissement dont les commentaires négatifs n'ont pas l'apanage et qui favorise sans doute la diffusion de l'ouvrage auprès du public autrichien. Certains critiques bienveillants expliquent, par exemple, que l'Église catholique n'est nullement visée $^{39}$. Or, le catholicisme est consubstantiel à l'Autriche et son empreinte sociale, omniprésente. B. Frischmuth aurait manqué son but si, désireuse d'enfoncer un coin dans la cohérence coercitive de l'éducation reçue dans l'après-guerre par nombre de jeunes Autrichiennes, elle avait négligé de prendre pour cible la composante catholique de cette construction langagière. Peut-on, du reste, supposer un instant une telle mansuétude dans un récit intitulé Die Klosterschule? Aveuglement ou mauvaise foi, peu importe: l'un comme l'autre sont significatifs. Le texte, lui, dit clairement dans le dernier chapitre la collusion entre les institutions catholiques et l'autorité, quelle qu'elle soit :

Jedermann sei der Obrigkeit untertan! Denn es gibt keine Gewalt, die nicht von Gott stammt. Wo immer eine besteht, ist sie von Gott angeordnet. Wer sich also gegen die Obrigkeit auflehnt, lehnt sich gegen die Anordnung Gottes auf ! Und zeigt uns nicht unser kath. Glaube den Sinn und das Ziel des Lebens, denn er allein macht selig, und darin ist doch alles enthalten usw. ${ }^{40}$

Le tour général de ce passage fait en outre surgir entre les lignes le passé récent de l'Autriche, tout comme le mot Gemeinschaft, présent dans le titre de la section $9^{41}$, n'est pas sans évoquer une idéologie de sinistre mémoire. L'écriture de B. Frischmuth est certes bien éloignée des imprécations et des raccourcis de Th. Bernhard qui, dans Die Ursache $^{42}$, établit un parallèle entre catholicisme et fascisme ; toutefois, escamoter la critique de l'Église catholique dans le texte de la première nous parait difficilement recevable.

\section{Des rêves de réalité assemblés au petit bonheur}

Il n'en demeure pas moins que, si l'interprétation édulcore souvent le contenu de Die Klosterschule, c'est parce que la tactique de la mimésis ne porte pas tous ses fruits. Le rêve nocturne qui, au cœur de l'ouvrage, inscrit plus lisiblement la rébellion de la narratrice, demeure lui aussi englué dans le discours à combattre. D'emblée, il est entaché de passivité, comme le souligne une formule introductive difficile à rendre en français ${ }^{43}$, que B. Frischmuth commente ainsi dans ses conférences de poétique :

Wieso wissen wir, daß wir träumen? Wissen wir es wirklich? Im allgemeinen wissen wir es erst, wenn wir geträumt haben. Und so wird der Schlaftraum mit « mir hat geträumt » referiert, während «ich träume » wünschendes, waches Träumen bedeutet ${ }^{44}$. 
nocturne de la jeune élève du pensionnat ne parvient qu'à mettre à la forme négative les formules dont elle est abreuvée, comme le montrent le début du chapitre, mentionné plus haut, et, de manière exemplaire, les lignes suivantes :

[Und es war] als existierten ich und die Welt und wären in schönster Ordnung und ganz gut so. Und wiederum, als existierten ich und die Welt nicht und wären nicht in schönster Ordnung und nicht ganz gut so ${ }^{45}$.

Quelques phrases plus loin, l'allusion à l'Alice de Lewis Carroll ${ }^{46}$, auquel B. Frischmuth voue une grande admiration ${ }^{47}$, suggère que le rêve joue le rôle d'un miroir qui restitue, inversées, les données de l'univers du pensionnat ; évoqués au chapitre 3, les véritables miroirs se refusent obstinément, quant à eux, à réfléchir la moindre parcelle d'individualité, la moindre féminitée ${ }^{48}$. Mais, ainsi que le note L. Irigaray, la traversée du miroir, simple renversement ${ }^{49}$, ne permet pas de se déprendre de la logique que l'on dénonce ; pour établir le lieu du féminin dans le discours, il faut en quelque sorte que le miroir devienne poreux, que la fluidité d'un incessant passage permette de déborder les oppositions :

Comment, donc, essayer de définir [...] ce travail du langage qui laisserait lieu au féminin? Disons que toute coupure dichotomisante, et redoublante à la fois - y compris entre énonciation et énoncé -, doit être déconcertée. Rien n'être plus jamais posé qui ne soit renversé, et renvoyé aussi à l'en-plus de ce renversement. Dit autrement : il n'y aurait plus ni endroit ni envers du discours, ni même du texte, mais les deux passant de l'un à l'autre pour faire « entendre » aussi ce qui résiste à cette structure recto-verso qui étaye le bon sens ${ }^{50}$.

De fait, le rêve de Die Klosterschule ne débouche que sur l'angoisse et la révolte impuissante suscitées par un appel divin tonitruant et incompréhensible :

Da schwoll die Stimme zu einem Lärm an, so gewaltig, daß ich nichts mehr verstehen konnte, ganz und gar nichts. Mir war gleich, als würde mir übel. [...] Wer nicht glaubt, wird nicht selig, aber wer soll denn glauben, bei dem Lärm da, ohne daß man zu Wort kommt, da könnte ja jeder sagen : komm ! - ${ }^{51}$

Dans le texte de B. Frischmuth se manifeste en vérité une démarche qui caractérise tous les écrivains du Forum Stadtpark à leurs débuts et dont on leur a parfois fait reproche, une critique dirigée en premier lieu contre Alfred Kolleritsch, en sa qualité d'éditeur de la revue manuskripte : la production et la promotion d'une littérature qui, dans le fond comme dans la forme, demeure exclusivement liée à ce qu'elle nie ${ }^{52}$.

B. Frischmuth va sortir de cet enfermement en empruntant des voies qui s'esquissent déjà dans Die Klosterschule: celle du récit et celle du rêve - ou plutôt la piste d'un récit fécondé par le rêve. Un rêve éveillé, cette fois, une "littérature du rêve " que l'écrivaine, citant E. Lenk ${ }^{53}$ définit ainsi :

Das Ketzerische dieser Literatur ist die Darstellung des Schwankenden, Labilen, Lebendigen, mitten in einer vom Kult der Exaktheit besessenen Welt, die Darstellung von Wesen, deren äußere Erscheinungsform permanent wechselt, ohne daß irgendeine der Gestalten sich als die wahre, die richtige, identifizieren ließe. Jedes Teil lebt, existiert für sich, lebt ein autonomes Leben. Die hierarchische Wertordnung der Wachheit ist aufgehoben ${ }^{54}$.

25 L'évocation de ce tremblotement qui a raison des lignes nettes et des angles vifs d'une réalité donnée pour inébranlable nous paraît très proche de la manière dont L. Irigaray caractérise le «style » du féminin :

Ce «style ", ou " écriture ", de la femme met plutôt feu aux mots fétiches, aux termes propres, aux formes bien construites. Ce «style » ne privilégie pas le regard mais rend toute figure à sa naissance, aussi tactile. Elle s'y re-touche sans jamais y 
constituer, s'y constituer en quelque unité. La simultanéité serait son " propre ». Un propre qui ne s'arrête jamais dans la possible identité à soi d'aucune forme. Toujours fluide, sans oublier les caractères difficilement idéalisables de ceux-ci : ces frottements entre deux infiniment voisins qui font dynamique. Son « style » résiste à, et fait exploser, toute forme, figure, idée, concept, solidement établis ${ }^{55}$.

Figure de la fluidité, la métamorphose est au cœur de la Sternwieser-Trilogie (Trilogie d'Amaryllis Sternwieser), triptyque destiné aux adultes qui paraît dans la deuxième moitié des années soixante-dix ${ }^{56}$. La fée Amaryllis, inquiète de la domination destructrice que les humains exercent sur la nature sous les yeux impuissants des fées et des esprits qui, depuis les Lumières, ont renoncé par nonchalance à cultiver et à utiliser leurs facultés surnaturelles, décide d'embrasser la nature humaine, "afin de répartir le pouvoir de manière nouvelle $»^{57}$. Tout comme Amaryllis dans le monde des humains, le merveilleux s'invite dans l'écriture de B. Frischmuth, qui offre sa revanche à la Schéhérazade bâillonnée de Die Klosterschule. Sous le regard d'Amy Stern s'éveillant à l'humanité dans sa chambre d'étudiante s'inversent l'ici et l'ailleurs classiques du merveilleux. L'initiation n'est pas celle d'un humain aux mystères, mais celle d'une exfée inventant les gestes de notre quotidien. Le Huron de Voltaire est ici en jupon et a reçu en outre, grâce à son origine merveilleuse, l'imagination en partage: de quoi inventer des déclinaisons de la vie au féminin. La métamorphose d'Amaryllis permet de ne pas en rester au contre-modèle, mais de déployer l'éventail des possibles. "Comment viennent les idées? Quand on fait de l'imagination l'organe de la réflexion » ${ }^{58}$ affirme B. Frischmuth; en écho, sa fée aime à s'exclamer : «Par la profusion des choses et des formes! $»^{59}$. Cette libération de l'imagination a partie liée avec la grammaire du rêve dont l'écrivaine propose à la littérature de tirer profit:

Die Traumform als literarische Vorlage bietet vieles, etwa ein unbefangeneres Aneinanderreihen unterschiedlichster Einfälle im Vertrauen darauf, daß alles zusammenhängt ${ }^{60}$.

La collision des "rêves de réalité assemblés au petit bonheur " ${ }^{61}$ peut provoquer les étincelles capables d'«embraser les formes bien construites " pour faire émerger d'autres cohérences, étrangères et étranges.

Le choix du merveilleux, dans les années soixante-dix, suppose un certain courage: celui de renoncer à l'aura avant-gardiste qui nimbe les écrivains de Graz. Nous avons retracé ailleurs ${ }^{62}$ la manière dont cette option a été diversement reçue par la critique qui y voit souvent un tournant radical, sans bien saisir qu'il s'inscrit aussi dans la continuité d'une évolution commune à la plupart des écrivains du Forum Stadtpark, dont les textes prennent peu à peu un tour utopique ${ }^{63}$. Au-delà de cette tendance générale, l'attachement résolu et apparemment désuet au rêve ${ }^{64}$ caractérise l'écriture de B. Frischmuth. Elle oppose, dans Literatur des Traums, les recettes en nombre assez limité qui président selon elle à toute expérimentation langagière consciente, aux chocs des rapprochements inattendus dont le rêve est prodigue ${ }^{65}$.

Il est logique que les conférences de poétique de l'écrivaine fassent une place de choix $\mathrm{au}$ « rêve féminin », titre de la cinquième d'entre elles. L'engagement pour la cause des femmes en littérature y est aussi vigoureux que le refus de toute orthodoxie féministe ${ }^{66}$. B. Frischmuth développe, par exemple, un point de vue qui s'incarne déjà dans les personnages de Sophie, Amy et Miranda de la Sternwieser-Trilogie, à une époque où il est loin de faire l'unanimité chez artistes et écrivaines: celui d'une féminité créatrice qui non seulement n'exclut pas la maternité, mais y puise une part de ce qui nourrit la création. L'enfant, comme le note B. Frischmuth dans «Le rêve féminin », enrichit la 
sensualité où puise l'écriture; il est à ce titre lui-même créateur ${ }^{67}$. Aucune concession non plus envers un certain discours féministe qui voit dans le nazisme la forme monstrueusement exacerbée du patriarcat et dans les femmes sous ce régime, sans distinction, de doubles victimes. Die Klosterschule montrait déjà comment les femmes peuvent se faire le relais du discours qui les opprime et y chercher, dans certains cas, un alibi pour fuir leurs responsabilités devant les désordres du monde. Vingt ans plus tard, en concordance avec les réflexions que certaines féministes de langue allemande commencent à développer ${ }^{68}$, le roman Einander Kind (Enfant l'un de l'autre), troisième volet de la Demeter-Trilogie (La trilogie de Déméter) ${ }^{69}$, met en scène en la personne de l'épouse du patriarche Schwarz et de sa fille Marie la culpabilité de certaines femmes sous le Troisième Reich. Dans la quatrième conférence de Traum der Literatur,B. Frischmuth évoque, à propos de ce récit, la génération des mères soumises, des mères coupables, des mères coupables parce que soumises; elle explique comment elle a cherché dans le mythe de Déméter et de Perséphone, divinités indissociables, une clé pour comprendre et parvenir à accepter le lien qui unit mères et filles, par-delà la culpabilité :

Konnte das nicht auch ein Hinweis darauf sein, daß man sich immer ähnlicher wurde, Mutter und Tochter, Tante und Nichte, oder vielmehr, daß man in Wirklichkeit gar nicht so verschieden war voneinander, wie man gerne geglaubt hätte? Vor allem meine Generation, die, mit den kriegführenden Vätern überworfen, den großteils schweigenden oder schweigend einverständigen Müttern, ob sie sich nun persönlich schuldig gemacht hatten oder nicht, alles nur nicht gleichen wollte ${ }^{70}$.

30 Faire du merveilleux et du mythe les ressorts d'une écriture désireuse d'inventer une réalité sociale qui permettrait enfin aux femmes de rester fidèles à toutes les composantes de leur personnalité est sans doute risqué, et les résultats de cette tentative ont été diversement appréciés par la critique féminine. Mais quel que soit le regard que l'on porte sur eux, l'on ne peut nier que la démarche possède le panache de l'indépendance et l'intelligence de l'auto-ironie, qui protège de tout monolithisme :

Sich endlich über die eigenen Strategien und Trugschlüsse lustig machen, ohne der Angst anheimzufallen, man liefere dem Gegner nur wieder Argumente, eine Angst, die genauso schlimm ist wie Selbstzensur aus politischen Rücksichten. Denken, was nicht sein darf, und was nicht ist, erfinden ${ }^{71}$ !

Tel est le souhait formulé par B. Frischmuth.

\section{NOTES}

1. «Da träumte es mir, ich wäre ein Licht - und jemand stellte seinen Scheffel darüber» (Barbara Frischmuth, Die Klosterschule, Reinbek bei Hamburg, rororo, 2006, p. 43). Dans la suite, nous citerons cette édition dans l'orthographe originale et sous la forme abrégée $K$, suivie du numéro de page. Aucun des textes de B. Frischmuth n'est paru en français.

2. Barbara Frischmuth, Die Klosterschule, Frankfurt am Main, Suhrkamp, 1968.

3. «Ihr seid das Licht der Welt. [...] Man zündet [...] nicht ein Licht an und stülpt ein Gefäß darüber, sondern man stellt es auf den Leuchter, dann leuchtet es allen im Haus» (Matthäus, 5, 
14-15, Die Heilige Schrift. Einheitsübersetzung, Stuttgart, Verlag Katholisches Bibelwerk/ Deutsche Bibelgesellschaft, 1998, p. 1345) ; "Vous êtes la lumière du monde. [...] Quand on allume une lampe, ce n'est pas pour la mettre sous le boisseau, mais sur son support et elle brille pour tous ceux qui sont dans la maison" (Évangile selon Saint Matthieu, 5, 14-15, Nouveau Testament. TOB, Paris, Éditions du Cerf, 1972, p. 53).

4. Kurt Bartsch, «Damals vor Graz: Die verspätete Aneignung von Moderne und Avantgarde in der Literatur aus dem Umkreis von Forum Stadtpark Graz und der Zeitchrift manuskripte», Études Germaniques, avril-juin 1995, 50e année, $\mathrm{n}^{\circ} 2$, p. 288.

5. Cf. Hugo vonHofmannsthal, «Die Idee Europa» (1917) et «Die österreichische Idee» (1917), in : Gesammelte Werke, Bd. II : Reden und Aufsätze 1914-1924, Frankfurt am Main, Fischer Taschenbuch Verlag, 1979, p. 43-54 et p. 454-458.

6. Cette attitude se dessine avant même que l'Autriche ne recouvre sa souveraineté, comme le montre un sondage réalisé par Der Turm auprès de plusieurs écrivains dès 1946. La question posée est la suivante : «Was ist österreichisch?» L'éditeur de la revue écarte d'emblée toute définition qui se référerait à un passé nationaliste et raciste, et précise : «[die Frage] bestimmt [...] sich ihrem Wesen nach als eine geistige Frage. Österreichisch - das ist ein geistiger Wesenszug, den die Geschichte und das Schicksal geformt haben. Es ist [...] ein Bekenntnis zum Geist als der formenden Kraft unseres Daseins». Les réponses formulées constituent un avatar de l'idéal hofmannsthalien, qui perd dans la crise de l'après-guerre encore un peu de sa substance ( $c f$. Albert Berger, "Zur Funktion des Begriffs der "österreichischen Literatur"», in : Sigurd Paul Scheichlet Gerald Stieg (éds.), Österreichische Literatur des 20.Jahrhunderts. Französische und österreichische Beiträge, Innsbruck, Institut für Germanistik der Universität Innsbruck, 1986, pp. 27 -28).

7. Max Mell (1951), Franz Nabl (1953), Paul Anton Keller (1955), Bruno Brehm (1962) ou encore Josef Papesch (1963) se voient ainsi honorés (cf. Bartsch, «Damals vor Graz», op. cit., p. 292-293).

8. Gunter Falk et Alfred Kolleritsch, «marginalie», manuskripte, 1964, $4^{\mathrm{e}}$ année, n¹2, p. 1. Cité dans Kurt Bartsch, «Das Forum Stadtpark Graz und seine Zeitschrift manuskripte in den 1960er Jahren. Eine Avant-Garde?», in : Kurt Bartsch et Gerhard Melzer (éds), Trans-Garde. Die Literatur der « Grazer Gruppe ». Forum Stadtpark und manuskripte, Graz, Droschl, 1990, p. 12.

9. «Das Programm ist auch offen für Avantgardistisches und Experimentelles, insgesamt selbst alles andere als avantgardistisch» (Bartsch, «Damals vor Graz», op. cit., p. 294).

10. "Ora et labora», «Spazierengehen», «Die Art der Betrachtung», «Schimäre», «Das Schulbeispiel», «Die Anstandsstunde», «Der Traum», «Das Glück», «Das Wesen der Gemeinschaft», «Antonius und Kleopatra», «Religionsunterricht», «Das Fleisch und das Blut», «Der Geist und das Fleisch», «Der Lauf der Welt».

11. K 42.

12. K 50 .

13. Nous formulons une hypothèse. Toutefois, si elle se tient, la discrétion de la dénonciation est ici peut-être excessive, puisque aucun interprète, à notre connaissance, ne s'est interrogé sur la division du texte en quatorze sections.

14. $\mathrm{K} 7$.

15. «Und so beten wir denn, bis uns geholfen wird» (K 9).

16. Klaus Zeyringer, Österreichische Literatur seit 1945, Innsbruck, Haymon-Verlag, 2001, p. 154.

17. Thomas Rothschild, «In die entgegengesetzte Richtung. Barbara Frischmuth: Die Klosterschule und Thomas Bernhard: Die Ursache», in : Silvana Cimenti et Ingrid Spörk, Barbara Frischmuth, Graz, Droschl (« Dossier extra »), 2007, p. 88.

18. «Es ist uns verboten worden, am Wochenende zu den Eltern nach Hause zu fahren. Man hat uns dabei ertappt, wie wir abends im Schlafsaal Geschichten erzählten, als das Licht nicht mehr brannte. Zu gewissen Zeiten ist es verboten, Geschichten zu erzählen - und die Nacht ist eine gewisse Zeit» (K 47, c'est nous qui soulignons). 
19. Les Mille et Une Nuits. I. Nuits 1 à 327, texte traduit, présenté et annoté par Jamel Eddine Bencheikh et André Miquel, Paris, Gallimard nrf, coll. « Bibliothèque de la Pléiade », 2005, p. 12.

20. Barbara Frischmuth, Traum der Literatur. Literatur des Traums. Münchner Poetik-Vorlesungen, Salzburg/ Wien, Residenz, 1991, p. 11.

21. Luce Irigaray, Ce sexe qui n'en est pas un, Paris, Éd. de Minuit, 1977, p. 73. Die Klosterschule anticipe, en quelque sorte, certaines des analyses de L. Irigaray. B. Frischmuth la citera du reste dans Traum der Literatur (op. cit., p. 73), preuve que leurs préoccupations se rejoignent.

22. Ibid., p. 73-74. C'est L. Irigaray qui souligne.

23. Cf. par exemple Peter Bauk, «Aufstand im Paradiesgärtlein», Stuttgarter Zeitung (Stuttgart), 4 mai 1968 ou Dietlind Kasarek, «Was ist und was sein könnte. Neue Prosa von Barbara Frischmuth», Badische Zeitung (Freiburg im Breisgau), 4 mars 1972.

24. Uwe Stamer, «Unterm Internats-Deckmantel. Zum Wiedererscheinen von Barbara Frischmuths Klosterschule», Stuttgarter Zeitung (Stuttgart), 23 déc. 1978.

25. Ibid.

26. «[H]ier, im letzten Kapitel, meldet sich Zweifel, zaghafter Widerstand, Revolte. Die Erzählerin entwickelt zum ersten Mal "eine Art Widerwillen gegen die zahllosen Gebete"» [K 88] [...] Das erzählende [...] Subjekt in Barbara Frischmuths Klosterschule steht nicht von vornherein in Opposition zur Institution» (Rothschild, «In die entgegengesetzte Richtung», op. cit., p. 88 et 89). Les remarques du critique Ruediger Engerth vont dans le même sens : «Die Klosterschule ist im Grunde ein gemütliches Buch, das mehr an eine Maturazeitung erinnert als an eine erbitterte Auseinandersetzung mit einem Erziehungssystem» («Aus einer Klosterschule», A.Z. Tagblatt für Ö sterreich (Wien), 8 avril 1979).

27. K 88 .

28. Rothschild, «In die entgegengesetzte Richtung», op. cit., p. 88.

29. Th. Rothschild est du reste contraint par l'examen du texte d'en convenir lui-même (ibid., p. 89).

30. «Tout se passe [...] comme si l'emploi du présent, en rapprochant [histoire et narration], avait pour effet de rompre leur équilibre et de permettre à l'ensemble du récit, selon le plus léger déplacement d'accent, de basculer soit du côté de l'histoire, soit du côté de la narration, c'est-àdire du discours » (Gérard Genette, Figures III, Paris, Seuil, 1972, p. 231).

31. «Bibelzitate, Merksätze, Passagen im drohenden Ton der Hausordnung, Auswendiggelerntes [...] - all das bäckt Barbara Frischmuth zu einem Sprachstil zusammen, aus dessen Verlautbarungen alles Persönliche getilgt ist. Siebzig Seiten Sprachmimikry, die rasch ihren Reiz verlieren. [...] Auf das Geständnis der Unfreiheit am Schluß ist das Werk angelegt. Doch: triumphiert hier nicht Ideologie über gestalterische Kraft? Haben nicht die Kapitel vorher verkrüppeltes Denken, gebrochenen Lebenswillen durch ironische Sprachspielereien und verschleierte Bekenntnisse zum eigenen Ich mit feinerem Stilgefühl ausgedrückt?» (Rolf Michaelis, «Namen, die man sich merken muß?», FAZ (Frankfurt a. Main), 12 févr. 1968. C'est nous qui soulignons).

32. «Die Klosterschule, bestehend aus 14 locker verbundenen Reflektionen und Schilderungen einer Schülerin, weder parodistisch noch ironisch gehalten, langweilt mehr als daß sie [...] aufklärt», déclare H. L. Arnold. Mais, citant le début du chapitre «Religionsunterricht», il affirme ensuite : «Ich meine, dieses Zitat bezeichnet das gesamte Verfahren von Barbara Frischmuth ziemlich genau. Und das soll weder Parodie noch Ironie enthalten? Ich neige fast zu dieser Annahme, obgleich das Parodistische und Ironische wenig kunstvoll auftritt» (Heinz Ludwig Arnold, «Zu Die Klosterschule», Deutsches Allgemeines Sonntagsblatt, 31 mars 1968, in : Kurt Bartsch (éd.), Barbara Frischmuth, Graz / Wien, Droschl, 1992, p. 111. C'est nous qui soulignons).

33. «Wohl wünscht man sich [...] ein auch sprachlich offeneres Bekenntnis - dort, wo die Ablehnung eindeutig ist -, doch wird man die Ursache für ein solches Zögern in der Scheu vor der definitiven Negation, geschähe sie auch unter dem Deckmantel des fiktionalen Ichs, zu suchen haben» (Stamer, «Unterm Internats-Deckmantel», op. cit.). 
34. Ce qui se traduisit une fois par le refus des sœurs de délivrer à leur élève le certificat d'excellence (Vorzugszeugnis) pourtant déjà établi (cf. Elisabeth Welzig, «Trobadora Barbara», Profil (Wien), n³8, 15 sept. 1980).

35. Cf. anon., «Heftige Reaktionen bei Literaturgespräch», Welser Zeitung (Wels), 12 nov. 1970.

36. «Nur ergibt sich ein kleiner Nachteil für [B. Frichmuth] aus dem Umstand, daß der Bedeutungsgrad des Themas bei ihr biographisch und subjektiv bedingt ist» (Bauk, «Aufstand im Paradiesgärtlein», op. cit.).

37. Roland Barthes, "L'effet de réel ", in: Roland Barthes, L. Bersani, philippe Hamonet al., Littérature et réalité, Paris, Seuil, 1982, p. 81-90.

38. Reinhard Priessnitz et Mechthild Rausch, «tribut an die tradition. aspekte einer postexperimentellen literatur», in : Peter Laemmle et Jörg Drews, Wie die Grazer auszogen, die Literatur $z u$ erobern. Texte, Porträts, Analysen und Dokumente junger österreichischer Autoren, München, dtv, 1979, p. 132.

39. «[Die] Klosterschule [...] [stellt] keineswegs einen Angriff auf die katholische Kirche dar [...], wie manche meinen, sondern [greift] jene autoritätsgläubige Erziehung an, wie sie in fast allen Internaten jeglicher Färbung, militärischer Anstalten und Schulen üblich ist» (anon., «Heftige Reaktionen bei Literaturgespräch», op. cit.).

40. K 88 .

41. «Das Wesen der Gemeinschaft» (K 51).

42. Thomas Bernhard, Die Ursache. Eine Andeutung, Salzburg, Residenz, 1975.

43. Cf. supra, note 1 .

44. Frischmuth, Traum der Literatur, op. cit., p. 19.

45. K 43.

46. «Christus (oder Gottvater) aber rief laut: Komm! Lieber nicht, schrie ich und wurde erheblich kleiner. Christus (oder Gottvater) aber rief laut: Komm! Da wurde ich wieder unerheblich größer [...]» (K. 44. C'est nous qui soulignons).

47. Cf. Frischmuth, Traum der Literatur, op. cit., p. 41-42.Parmi les nombreux récits pour enfants dont B. Frischmuth est l'auteur, se trouve un Alice im Wunderland (Berlin, Aufbau Verlag, 2000).

48. Cette section baptisée «Die Art der Betrachtung» (K 20-23) propose par exemple une description purement géométrique de l'image du visage de la narratrice, dont les traits s'effacent sous les précisions mathématiques: «Wenn ich meinen Blick als den Ort auffasse, der sich zu einer Waagrechten verlängern läßt, so beginnt fünf Fingerbreit über dem Blick mein Haar. Vier Fingerbreit unter dem Blick endet meine Nase. Zwei Fingerbreit unter dem Punkt, an dem meine Nase endet, liegt parallel zu meinem Blick mein Mund [...]» (K 22). Le chapitre se clôt sur la phrase : «Ansonsten fällt mir an meinem Gesicht nichts auf» (K 23).

49. Dans la « Maison du Miroir » que décrit Alice à Kitty, « les objets [...] sont inversés » et dans les livres, "les mots [...] sont écrits à l'envers" (Lewis Carroll, De l'autre côté du miroir et de ce qu'Alice y trouva, in: Lewis Carroll, Tout Alice, trad. de l'anglais par Henri Parisot, Paris, Flammarion, 1979, p. 217).

50. . - Irigaray, Ce sexe qui n'en est pas un, op. cit., p. 76-77. C'est L. Irigaray qui souligne.

51. K. 44-45 et 46.

52. «Elisabeth Wiesmayr [...] kritisiert, [...] [n]egativ sei Kolleritsch [...] selbst den Positionen verhaftet, die er anprangert» (Bartsch, «Damals vor Graz», op. cit., p. 293-294).

53. Elisabeth Lenk, Die unbewusste Gesellschaft, Matthes und Seitz, München, 1983.

54. Frischmuth, Traum der Literatur, op. cit., p. 48.

55. Irigaray, Ce sexe qui n'en est pas un, op. cit., p. 76.

56. Il se compose desromans Die Mystifikationen der Sophie Silber (1976), Amy oder Die Metamorphose (1978) et Kai und die Liebe zu den Modellen (1979). Pour ne pas excéder les limites qui nous sont imparties ici, nous nous bornons à retracer à grands traits l'évolution de l'écriture de 
B. Frischmuth et ne mentionnons à cette fin que les textes qui nous semblent le mieux en refléter la nature.

57. «Wir können [...] die Gestalt ihrer Frauen und Kinder annehmen und die Macht neu verteilen» (Barbara Frischmuth, Die Mystifikationen der Sophie Silber, Berlin, Aufbau Taschenbuch Verlag, p. 313).

58. «Wie kommt es zu Ideen? Indem man mit seiner Phantasie nachdenkt» (Barbara Frischmuth, «Der Ort der Phantasie», Die Presse (Wien), 9-10 juil. 1977).

59. «Bei allen Dingen und Gestalten!».

60. Frischmuth, Traum der Literatur, op. cit., p. 41.

61. Cette formule estle titre d'un article d'Ulf Heise : «Zusammengewürfelte Wirklichkeitsträume. Auch sie ist von Wittgenstein traktiert: die Österreicherin Barbara Frischmuth», Neue Zeit (Berlin), 6 juil. 1991.

62. Hélène Barrière, «L'apparition du merveilleux dans l'écriture de Barbara Frischmuth : les enjeux de la métamorphose", communication au colloque "Le merveilleux, entre mythe et religion» organisé par Anne Besson et Évelyne Jacquelin, Arras, 25-26 septembre 2008. À paraître.

63. «[D]ie Literatur der « Grazer » [gibt] die einseitige Bindung an das Negierte allmählich auf [...] und [akzentuiert] Dimensionen des Utopischen [...]»(Gerhard Melzer, «Die Verlegenheitsgruppe. Zur Geschichte der "Grazer Literatur"», in : Bartsch et Melzer (éds), Trans-Garde, op. cit., p. 30).

64. «Den Traum und seine Mechanismen, seine Funktionsweisen, literarisch zu nutzen, hat lange Zeit methodisch und formal als der letzte Stand gegolten, als erschlossenes Neuland (oder ist es doch nur ganz altes, vergessenes Land?» (Traum der Literatur, op. cit., p. 47).

65. Ibid., p. 46-47.

66. Une étiquette que B. Frischmuth ne revendique pas, bien au contraire : «eigentlich bin ich keine militante Feministin... [...] ich mag alles nicht, was eine "istin" daran hat. [...] ich finde es lächerlich, weil das wieder eine Einordnung in eine Lade bedeutet» (Lori Ann Ingalsbe, «Ein Gespräch mit Barbara Frischmuth», Modern Austrian Literature, 1996, vol. 29, n¹, p. 48).

67. Frischmuth, Traum der Literatur, op. cit., p. 76-77.

68. $C f$. par exemple: AG Frauen gegen den Antisemitismus, «Der Nationalsozialismus als Extremform des Patriarchats. Zur Leugnung der Täterschaft von Frauen und zur Tabuisierung des Antisemitismus in der Auseinandersetzung mit dem NS», beiträge zur feministischen theorie und praxis, 1993, 16e année, vol. 35 «Feminis-muß», p. 77-89.

69. Elle comprend les romans : Herrin der Tiere (1986), Über die Verhältnisse (1987) et Einander Kind (1990).

70. Frischmuth, Traum der Literatur, op. cit., p. 70-71.

71. Ibid., p. 72-73.

\section{RÉSUMÉS}

Die Klosterschule (1968), premier roman de l'Autrichienne B. Frischmuth, reflète les préoccupations du Forum Stadtpark de Graz, dont elle a été en 1960 l'un des membres fondateurs. Né en réaction au refoulement du passé nazi et au culte de la tradition qui prévalent dans l'Autriche de l'après-guerre, le Forum Stadtpark voit dans le langage l'instrument de formatage des individus grâce auquel se perpétue une forme de société - mécanisme à enrayer par 
l'expérimentation sur la langue. Dans Die Klosterschule, citation et montage, juxtaposition de bribes du quotidien, asphyxie du « je » narratif mettent au jour les mécanismes langagiers à l'aide desquels, dans les internats catholiques de filles, l'on inculque à celles-ci la soumission aux structures patriarcales. Mais cette appropriation légèrement décalée du discours oppressif afin de le déjouer, que L. Irigaray nommera plus tard stratégie du mimétisme, n'est pas toujours perçue dans toute son ampleur dénonciatrice, comme le montre la réception du roman. Dans les années 80 et 90, B. Frischmuth, comme d'autres écrivains du Forum Stadtpark décriés par les tenants de l'avant-garde expérimentale, quitte le registre de la pure dénonciation pour celui de l'utopie: à l'aide de modes d'écriture plus traditionnels (merveilleux, mythe), elle tente d'esquisser des déclinaisons inédites de la vie au féminin. Cette "littérature du rêve", diversement appréciée par les féministes, manifeste toutefois le courage de l'indépendance et l'intelligence de l'auto-ironie.

In der Klosterschule (1968), dem ersten Roman der Österreicherin B. Frischmuth, schlägt sich eine Einstellung zur Sprache nieder, die den frühen Texten der Autoren von Forum Stadtpark Graz gemeinsam ist, zu dessen Gründungsmitgliedern B. Frischmuth 1960 zählte. Als Protest gegen die im Nachkriegsösterreich vorherrschende Vergangenheitsverdrängung und Huldigung der Tradition entstanden, denunziert Forum Stadtpark die Disziplinierung durch und in Sprache als Mittel, mit dem eine Gesellschaftsform ihr Fortbestehen sichere und welches es durch sprachanalytische Strategien zu bekämpfen gelte. In der Klosterschule bringen Zitat und Montage, Aneinanderreihung von Alltagsbruchstücken, ein im „Wir“ der Gemeinschaft eingeklemmtes erzählendes Ich Sprachmechanismen ans Licht, durch die im katholischen Internat den Mädchen widerstandsloses Hinnehmen patriarchalischer Herrschaftsverhältnisse anerzogen wird. Doch die Aufnahme der Klosterschule zeigt, dass die kritische Virulenz dieser ironischen, später von L. Irigaray als Strategie der Mimikry bezeichneten Aneignung des Unterdrückungsdiskurses, nicht in ihrem ganzen Umfang erkannt wird. In den 80er und 90er Jahren gibt B. Frischmuth, wie andere, manchmal als Verräter an der Avantgarde angeprangerte „Grazer“, die einseitige Bindung an das Negierte auf, um Dimensionen des Utopischen zu akzentuieren. Durch Zugriff zu traditionelleren Schreibweisen (Wunderbares, Mythos) versucht sie, neue weibliche Entfaltungsmöglichkeiten zu skizzieren. In dieser von den Feministen divers beurteilten „Literatur des Traums“ bekundet sich B. Frischmuths Mut zur Unabhängigkeit und erfrischende Selbstironie.

Die Klosterschule (1968), the first novel of the Austrian authoress B. Frischmuth, reflects concerns of Graz's Forum Stadtpark the foundation of wich she contributed in 1960. Born in reaction to traditions and the suppression of Nazi times in post-war Austria, Forum Stadtpark conceives of language as the main tool for formatting individuals into a kind which society can immortalize : this mechanism must be opposed by means of language experiment. In Die Klosterschule, quotation and editing, juxtaposition of snatches of everyday life, and suffocation of the narrative "I" reveal language machinery with which girls are taught to submit to patriarchal hierarchies in catholic girls' boarding schools. Nonetheless this slightly staggered use of oppressive discourse in order to foil it - that L. Irigaray will later call "mimicry strategy" - is not often seen as it should be (i.e. a large-scale criticism), as is shown by novel reception shows it. In the 80 's and the 90's, B. Frischmuth follows the way of other Forum Stadtpark's writers who were denied by avant-garde literature : she uses utopia instead of writings of pure denunciation. Applying more traditional literary genres (supernatural novels, myth), she attempts to present a range of unusual female lifestyles. This "dream literature", variously appreciated by feminists, reveals courageous independance and intelligent self-irony. 
AUTEUR

HÉLĖNE BARRIÈRE

Université de Provence 